\title{
Instabilities of a Time-Dependent Shear Flow
}

\author{
TIMOUR RADKO \\ Department of Oceanography, Naval Postgraduate School, Monterey, California
}

(Manuscript received 13 March 2019, in final form 13 June 2019)

\begin{abstract}
This study offers a systematic stability analysis of unsteady shear flows representing large-scale, lowfrequency internal waves in the ocean. The analysis is based on the unbounded time-dependent Couette model. This setup makes it possible to isolate the instabilities caused by uniform shear from those that can be attributed to resonant triad interactions or to the presence of inflection points in vertical velocity profiles. Linear analysis suggests that time-dependent spatially uniform shears are unstable regardless of the Richardson number (Ri). However, the growth rate of instability monotonically decreases with increasing $\mathrm{Ri}$ and increases with increasing frequency of oscillations. Therefore, models assuming a steady basic state-which are commonly used to conceptualize shear-induced instability and mixing — can be viewed as singular limits of the corresponding timedependent systems. The present investigation is focused on the supercritical range of Richardson numbers $(\mathrm{Ri}>1 / 4)$ where steady parallel flows are stable. An explicit relation is proposed for the growth rate of shear instability as a function of background parameters. For moderately supercritical Richardson numbers $(\mathrm{Ri} \sim 1)$, we find that the growth rates obtained are less than, but comparable to, those expected for Kelvin-Helmholtz instabilities of steady shears at $\mathrm{Ri}<1 / 4$. Hence, we conclude that the instability of timedependent flows could represent a viable mixing mechanism in the ocean, particular in regions characterized by relatively weak wave activity and predominantly supercritical large-scale shears.
\end{abstract}

\section{Introduction}

Shear-induced destabilization of laminar currents and the subsequent generation of small-scale turbulence represents one of the major drivers of diapycnal mixing in the ocean interior (e.g., Smyth and Moum 2012). Motivated by numerous geophysical applications, the problem of instability of vertically sheared parallel flows has long evolved into a broad and active research area of fluid dynamics. Comprehensive reviews of the field can be found in, for example, Peltier and Caulfield (2003), Thorpe (2005), and Ivey et al. (2008). The following discussion is therefore focused on a small subset of results that are directly relevant for the present investigation.

Intermittent mixing events in the ocean interior are commonly conceptualized by assuming that the onset of turbulence is triggered by the well-known KelvinHelmholtz instability (KHI). KHI (von Helmholtz 1868; Kelvin 1871) occurs in stably stratified (i.e., with density increasing downward) unidirectional steady shear flows. The susceptibility of shear flows to KHI is traditionally determined using the Richardson number

Corresponding author: Timour Radko, tradko@nps.edu

$$
\mathrm{Ri}=\frac{-\frac{g}{\bar{\rho}^{*}} \frac{\partial \bar{\rho}^{*}}{\partial z^{*}}}{\left(\frac{\partial \bar{u}^{*}}{\partial z^{*}}\right)^{2}},
$$

where $\bar{\rho}^{*}\left(z^{*}\right)$ and $\bar{u}^{*}\left(z^{*}\right)$ are the vertical profiles of density and velocity in the basic state and asterisks hereafter denote dimensional field variables. Nondissipative steady shears are stable as long as the Richardson number exceeds the critical value of $\mathrm{Ri}_{\mathrm{cr}}=$ 1/4 (Richardson 1920; Miles 1961; Howard 1961). In the subcritical regime $(\mathrm{Ri}<1 / 4)$, nonlinear evolution of growing modes is typically characterized by a series of secondary instabilities, which ultimately generate fully turbulent billows-a scenario supported by observational (Woods 1968; van Haren et al. 2014), laboratory (Thorpe 1971; Atsavapranee and Gharib 1997), and numerical (Caulfield and Peltier 2000; Smyth et al. 2001) studies.

The inclusion of dissipative effects can substantially modify the conditions for-and the evolutionary patterns of-KHI. For instance, the critical Richardson number required for instability is sensitive to the assumed diffusivity and viscosity values. Somewhat counterintuitively, both viscous and diffusive effects could 
be either stabilizing or destabilizing, depending on a particular system (e.g., Balmforth and Young 2002; Thorpe et al. 2013). A recent numerical study of the KHI evolution in the field of preexisting turbulence revealed a tendency for suppression of the flow rollup relative to the patterns realized for corresponding laminar initial states (Kaminski and Smyth 2019). Another interesting effect is realized in two-component fluids with unequal diffusivities of density components, where weak, dynamically stable shears can destabilize the system by triggering so-called thermohaline-shear instability (Radko 2016).

In the oceanographic context, the vertical shear required for KHI is usually attributed to internal waves. The associated shears are, strictly speaking, neither unidirectional nor steady. However, since the spectrum of internal waves is dominated by relatively slow nearinertial waves (e.g., Garrett and Munk 1972), the steadystate approximation appears to offer a reasonable starting point for the analysis of shear-driven instabilities and it has been frequently used in the past (e.g., Dunkerton 1997). Nevertheless, the intrinsic timedependence of internal waves has some major consequences for their stability. A fundamentally different class of instabilities arises due to the possibility of resonant triad interactions between the primary internal wave and two low-amplitude harmonics. Several analyses of parametric subharmonic instability—usually the most rapidly amplifying type of instabilities induced by triad interactions-have led to a conclusion that propagating internal waves can be unstable regardless of its amplitude (Mied 1976; Drazin 1977; Klostermeyer 1982; Lombard and Riley 1996). This result appears to be robust; it is also supported by our analysis of timedependent shear instabilities (TDSI) in the Couette model. However, it should be kept in mind that the instability of a flow field does not always guarantee the transition to turbulence. For instance, the laboratory experiments of Troy and Koseff (2005) indicate that the threshold Richardson numbers for the formation of visible billows in oscillatory shears are actually significantly lower than the canonical value of $\mathrm{Ri}_{\mathrm{cr}}=1 / 4$.

The objective of the present study of time-dependent shear flows is threefold. First, we embark on a rather complete exploration of the parameter space in order to examine stabilizing/destabilizing effects of planetary rotation, temporal variability pattern of the background flow, and dissipation of buoyancy and momentum. Another defining feature of the present analysis is the chosen framework, which is based on the unbounded Couette flow. This configuration makes it possible to delineate the destabilization caused by uniform shear from (i) effects of resonant triad interactions between harmonic waves and (ii) instabilities associated with the presence of inflection points in vertical velocity profiles. In this regard, the present investigation can be related to the stability analyses of inertial currents by Winters (2008) and of oscillatory flows characterized by solid-body rotation in the vertical plane by Majda and Shefter (1998), although our methodology is substantially different. Finally, an attempt is made to identify parameter regimes where the growth rates are substantial and amplifying perturbations are thus likely to strongly influence the evolution of the flow field. An explicit scaling law is proposed, which captures the dependencies of growth rates on the characteristics of the basic flow.

The manuscript is organized as follows. The model configuration and the technique used in linear stability analyses are described in section 2. Section 3 explores properties of TDSI in a simplified two-dimensional framework. The physical interpretation of TDSI is offered in section 4, where the growth of unstable perturbations is linked to the strain-induced modulation of vertical velocity (the Orr mechanism). Section 5 presents the analysis of a more complicated and realistic three-dimensional system under the influence of planetary rotation. The results are summarized and conclusions are drawn in section 6 .

\section{Formulation}

The total density field $\rho^{*}$ is separated into the background component $\bar{\rho}^{*}$ and a small departure $\rho^{\prime *}$ from it:

$$
\rho^{*}=\bar{\rho}^{*}\left(z^{*}\right)+\rho^{*}\left(x^{*}, y^{*}, z^{*}, t^{*}\right),
$$

where asterisks denote dimensional quantities. We consider a linearly stratified basic state:

$$
\bar{\rho}^{*}=A_{\rho}^{*} z^{*}+A_{\rho 0}^{*},
$$

where $\left(A_{\rho}^{*}, A_{\rho 0}^{*}\right)$ are constants and $\partial \bar{\rho}^{*} / \partial z^{*}=A_{\rho}^{*}<0$.

The velocity field $\mathbf{v}^{*}=\left(u^{*}, v^{*}, w^{*}\right)$ is similarly separated into the basic state $\overline{\mathbf{v}}^{*}$, which is assumed to be time dependent, and a weak perturbation $\mathbf{v}^{\prime *}$. The horizontal components of basic velocity $\left(\bar{u}^{*}, \bar{v}^{*}\right)$ are finite and vary in $z^{*}$ and $t^{*}$. The basic vertical velocity $\bar{w}^{*}$ on the other hand is assumed to be negligible. This feature reflects the pattern of dominant internal waves in the ocean that operate at low (close to inertial) frequencies and are characterized by nearly horizontal wave fronts. We further assume that the basic horizontal velocity components vary linearly in $z^{*}$ :

$$
\bar{u}^{*}=A_{U}^{*}\left(t^{*}\right) z^{*}, \quad \bar{v}^{*}=A_{V}^{*}\left(t^{*}\right) z^{*}, \quad \bar{w}^{*}=0 .
$$


The nonlinearity of the equation of state and the compressibility of seawater are ignored, which reduces the governing Boussinesq equations of motion to

$$
\left\{\begin{array}{l}
\frac{\partial \rho^{\prime *}}{\partial t^{*}}+\mathbf{v}^{*} \cdot \nabla \rho^{*}+w^{*} \frac{\partial \bar{\rho}^{*}}{\partial z^{*}}=k_{\rho} \nabla^{2} \rho^{\prime *}, \\
\frac{\partial \mathbf{v}^{*}}{\partial t^{*}}+\mathbf{v}^{*} \cdot \nabla \mathbf{v}^{*}+\left(-f^{*} v^{*}, f^{*} u^{*}, 0\right)=-\frac{1}{\rho_{0}^{*}} \nabla p^{*}-g \frac{\rho^{\prime *}}{\rho_{0}^{*}} \mathbf{i}_{z}+\nu \nabla^{2} \mathbf{v}^{*}+\mathbf{F}^{*}, \\
\nabla \cdot \mathbf{v}^{*}=0
\end{array}\right.
$$

where $p^{*}$ is the pressure, $g$ is gravity, $f^{*}$ is the Coriolis parameter, $\mathbf{i}_{z}$ is the vertical unit vector, $\mathbf{F}^{*}$ is the externally imposed pressure gradient force maintaining the basic velocity $\overline{\mathbf{v}}^{*}, \nu$ is the molecular viscosity, and $k_{\rho}$ is the molecular diffusivity of density. Note that here we make no attempt to represent effects of unequal diffusivities of heat and salt-two major components of seawater density - thereby intentionally excluding from our analysis a set of processes collectively referred to as double-diffusive convection (Stern 1960; Radko 2013).
System (5) is nondimensionalized using microstructure scales, on which effects of molecular viscosity and dissipation play an order-one role. Thus, $d=\left[k_{\rho} \nu /\left(g / \rho_{0}^{*}\right)\left|\partial \bar{\rho}^{*} / \partial z^{*}\right|\right]^{1 / 4}, d^{2} / k_{\rho}, k_{\rho} / d, \rho_{0}^{*} \nu k_{\rho} / d^{2}$, and $\left|\partial \bar{\rho}^{*} / \partial z^{*}\right| d$ represent the units of length, time, velocity, pressure, and density perturbations, respectively (e.g., Radko 2013). The governing system (5) is first nondimensionalized and then linearized with respect to the basic state $(\bar{\rho}, \bar{p}, \overline{\mathbf{v}})$, resulting in

$$
\left\{\begin{array}{l}
\frac{\partial \rho^{\prime}}{\partial t}+A_{U} z \frac{\partial \rho^{\prime}}{\partial x}+A_{V} z \frac{\partial \rho^{\prime}}{\partial y}-w^{\prime}=\nabla^{2} \rho^{\prime} \\
\frac{\partial u^{\prime}}{\partial t}+A_{U} z \frac{\partial u^{\prime}}{\partial x}+A_{V} z \frac{\partial u^{\prime}}{\partial y}+w^{\prime} A_{U}-f v^{\prime}=\operatorname{Pr}\left(-\frac{\partial p^{\prime}}{\partial x}+\nabla^{2} u^{\prime}\right) \\
\frac{\partial v^{\prime}}{\partial t}+A_{U} z \frac{\partial v^{\prime}}{\partial x}+A_{V} z \frac{\partial v^{\prime}}{\partial y}+w^{\prime} A_{V}+f u^{\prime}=\operatorname{Pr}\left(-\frac{\partial p^{\prime}}{\partial y}+\nabla^{2} v^{\prime}\right) \\
\frac{\partial w^{\prime}}{\partial t}+A_{U} z \frac{\partial w^{\prime}}{\partial x}+A_{V} z \frac{\partial w^{\prime}}{\partial y}=\operatorname{Pr}\left(-\frac{\partial p^{\prime}}{\partial z}-\rho^{\prime}+\nabla^{2} w^{\prime}\right) \\
\frac{\partial u^{\prime}}{\partial x}+\frac{\partial v^{\prime}}{\partial y}+\frac{\partial w^{\prime}}{\partial z}=0
\end{array}\right.
$$

where $f=f^{*} d^{2} / k_{\rho}$ is the nondimensional Coriolis parameter and $\operatorname{Pr}=\nu / k_{\rho}$ is the Prandtl number. The pressure field can be inferred diagnostically from the divergence of the momentum equations, and therefore (6) effectively represents the closed set of prognostic equations for $\left(\rho^{\prime}, u^{\prime}, v^{\prime}, w^{\prime}\right)$. To simplify the interpretation of our nondimensional results in terms of relevant oceanic scales, we assume the following nominal dimensional values of governing parameters:

$$
\begin{aligned}
& N^{* 2} \sim 10^{-5} \mathrm{~s}^{-2}, \quad \rho_{0}^{*} \sim 10^{3} \mathrm{~kg} \mathrm{~m}^{-3}, \quad g \sim 10 \mathrm{~m} \mathrm{~s}^{-2}, \\
& f^{*} \sim 10^{-4} \mathrm{~s}^{-1}, \quad \nu \sim 10^{-6} \mathrm{~m}^{2} \mathrm{~s}^{-1}, \quad k_{\rho} \sim 10^{-7} \mathrm{~m}^{2} \mathrm{~s}^{-1},
\end{aligned}
$$

which suggest $d=0.01 \mathrm{~m}$. The key nondimensional numbers are evaluated accordingly:

$$
f=0.1, \quad \operatorname{Pr}=10, \quad N=\sqrt{\operatorname{Pr}}=3.16 .
$$

A complication arising in the stability analysis of (6) is associated with $z$ dependence of its coefficients $\left(A_{V} z, A_{U} z\right)$, and the problem is treated using techniques utilized in earlier studies of the unbounded Couette model (Knobloch 1984; Shepherd 1985; Radko 2019). The perturbation fields are represented by a superposition of plane-wave components

$\left(\rho^{\prime}, u^{\prime}, \boldsymbol{v}^{\prime}, w^{\prime}\right)=\operatorname{Re}[(\hat{\rho}, \hat{u}, \hat{v}, \hat{w}) \exp (i k x+i l y+i m z)]$, 
where $(\hat{\rho}, \hat{u}, \hat{v}, \hat{w})$ are time dependent. Importantly, the vertical wavenumber $m$ in the uniform shear model (e.g., Shepherd 1985) is also a function of time:

$$
m=m_{0}-B_{U}(t) k-B_{V}(t) l,
$$

where

$$
B_{U}=\int_{o}^{t} A_{U}\left(t^{\prime}\right) d t^{\prime}, \quad B_{V}=\int_{o}^{t} A_{V}\left(t^{\prime}\right) d t^{\prime} .
$$

For each mode (9), transformation (10) reduces the governing system to a set of ordinary differential equations (ODEs):

$$
\frac{d}{d t}\left(\begin{array}{c}
\hat{\rho} \\
\hat{u} \\
\hat{v} \\
\hat{w}
\end{array}\right)=\mathbf{M}\left(\begin{array}{c}
\hat{\rho} \\
\hat{u} \\
\hat{v} \\
\hat{w}
\end{array}\right)
$$

where the elements of $4 \times 4$ matrix $\mathbf{M}$ depend on the wavenumbers $\left(k, l, m_{0}\right)$, time $t$, instantaneous values of shear $\left(A_{U}, A_{V}\right)$, their temporal integrals $\left(B_{U}, B_{V}\right)$, and the Prandtl number (Pr).

\section{Two-dimensional model}

To gain insight into the stability properties of (6), we first explore a relatively simple nonrotating twodimensional $(x, z)$ version of the model, which is obtained by assuming $l=0, \hat{v}=0, f=0$, and $A_{V}=0$. In this regime, the governing equations can be simplified by introducing streamfunction $\psi$, such that $(u, w)=$ $(-\partial \psi / \partial z, \partial \psi / \partial x)$. In the vorticity-streamfunction form, system (6) reduces to

$$
\left\{\begin{array}{l}
\frac{\partial \rho^{\prime}}{\partial t}+A_{U} z \frac{\partial \rho^{\prime}}{\partial x}-\frac{\partial \psi^{\prime}}{\partial x}=\nabla^{2} \rho^{\prime} \\
\frac{\partial \nabla^{2} \psi^{\prime}}{\partial t}+A_{U} z \frac{\partial \nabla^{2} \psi^{\prime}}{\partial x}=\operatorname{Pr}\left(-\frac{\partial \rho^{\prime}}{\partial x}+\nabla^{4} \psi^{\prime}\right)
\end{array} .\right.
$$

A monochromatic pattern is assumed for the background shear:

$$
A_{U}=a_{U} \cos (\omega t)
$$

The shear strength is traditionally quantified using the Richardson number in (1). In particular, we introduce the Richardson number based on the mean shear variance

$$
\overline{\mathrm{Ri}}=\frac{-\frac{g}{\rho_{0}^{*}} \frac{\partial \bar{\rho}^{*}}{\partial z^{*}}}{\left[\left(\frac{\partial \bar{u}^{*}}{\partial z^{*}}\right)^{2}\right]_{t}},
$$

where the symbol $[\ldots]_{t}$ denotes temporal averaging, and the minimal Richardson number is

$$
\mathrm{Ri}_{\min }=\frac{-\frac{g}{\rho_{0}^{*}} \frac{\partial \bar{\rho}^{*}}{\partial z^{*}}}{\max \left[\left(\frac{\partial \bar{u}^{*}}{\partial z^{*}}\right)^{2}\right]} .
$$

Both mean and minimal Richardson numbers can be expressed as a function of shear amplitude $a_{U}$ in (14) as follows:

$$
\overline{\mathrm{Ri}}=\frac{2 \mathrm{Pr}}{a_{U}^{2}}, \quad \mathrm{Ri}_{\min }=\frac{\operatorname{Pr}}{a_{U}^{2}} .
$$

In terms of the mean Richardson number $(\overline{\mathrm{Ri}})$, the canonical condition for stability of parallel flows with respect to KHI amounts to $\overline{\mathrm{Ri}}=2 \mathrm{Ri}_{\text {min }}>2 \mathrm{Ri}_{\text {cr }}=1 / 2$ and therefore our investigation will specifically target this supercritical regime.

Solutions of the two-dimensional model (13) are sought in the form of plane waves $\left(\rho^{\prime}, \psi^{\prime}\right)=\operatorname{Re}[(\hat{\rho}, \hat{\psi}) \exp (i k x+$ $i m z)]$ and the counterpart of (12) reduces to

$$
\frac{d}{d t}\left(\begin{array}{c}
\hat{\rho} \\
\hat{\psi}
\end{array}\right)=\mathbf{M}_{2 D}\left(\begin{array}{c}
\hat{\rho} \\
\hat{\psi}
\end{array}\right),
$$

where $\mathbf{M}_{2 D}$ is a $2 \times 2$ matrix with elements that depend on ( $\left.k, m_{0}, t, A_{U}, B_{U}, \operatorname{Pr}\right)$. Stability properties of the ODE system in (18) are determined by integrating it in time using random complex initial conditions for $(\hat{\rho}, \hat{\psi})$. Since this system is linear, its solutions eventually become dominated by the fastest growing mode. The growth rate $\lambda_{r}$ is evaluated by introducing the quadratic norm that is based on the net perturbation energy, which reduces, in our nondimensional units, to

$$
e=\frac{\left(k^{2}+m^{2}\right)|\hat{\psi}|^{2}}{4}+\frac{\operatorname{Pr}|\hat{\rho}|^{2}}{4}
$$

The best linear fit to $(1 / 2) \ln (e)$ is used to determine $\lambda_{r}$ for each $\left(k, m_{0}\right)$.

A typical calculation of this nature is shown in Fig. 1, which was performed for $\left(\overline{\mathrm{Ri}}, \omega, k, m_{0}\right)=$ $\left(1,0.1,1.5 \times 10^{-4}, 10^{-2}\right)$. The inspection of the energy amplification pattern in Fig. 1 indicates that, after a brief initial adjustment period, the perturbation starts to grow in a nearly exponential manner. It is also apparent that the overall exponential growth is modulated on the temporal scale of $20 \pi$, which matches the periodicity of the basic shear $2 \pi / \omega$. The calculations analogous to that in Fig. 1 were repeated for a wide range of wavenumbers and the overall 


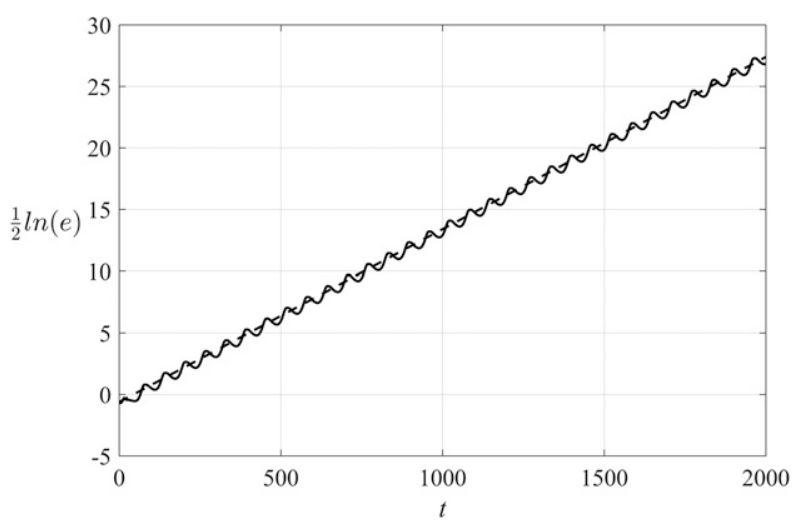

FIG. 1. The temporal record of $(1 / 2) \ln (e)$, where $e$ is the quadratic perturbation norm for $\overline{\mathrm{Ri}}=1, k=1.5 \times 10^{-4}, m_{0}=0.01$, and $\omega=0.1$. The overall amplification pattern can be closely approximated by a straight line (dashed), which implies that the perturbation grows at an exponential rate. However, the long-term exponential growth is modulated on small temporal scales with periodicity matching that of the basic state.

maximal growth rate $\lambda_{\max }$ was determined by maximizing $\lambda_{r}$ over the wavenumber space:

$$
\lambda_{\max }=\max _{k, m_{0}}\left(\lambda_{r}\right) .
$$

Because of the invariance of governing equations with respect to the transformation $\left(k, m_{0}\right) \rightarrow\left(-k,-m_{0}\right)$, the growth rate in (20) can be maximized over nonnegative values of $m_{0}$ without loss of generality.

The key objective of the following investigation is the analysis of the maximal growth rate as a function of $(\overline{\mathrm{Ri}}, \omega)$. The relevant range of $\omega$ could be estimated by recalling that the frequency of internal waves lies in the interval

$$
f<\omega<N,
$$

and their energy spectrum is dominated by near-inertial components $(\omega \sim f)$. Thus, even though planetary rotation is not incorporated in our two-dimensional calculations, we assume that, for baseline parameters in (8), the $\omega=0.1$ case represents the dominant (inertial) oscillations. It should also be kept in mind that the wave fronts of internal waves become progressively steeper with increasing frequency. Our model, on the other hand, is based entirely on the vertical shear of horizontal velocity and thus implicitly assumes that wave fronts are relatively flat. This implies that the proposed configuration offers a consistent representation of waveinduced shear for as long as $\omega \ll N \sim 3.16$. The following analysis is therefore restricted to the range $\omega<1$.

Figure 2 presents a series of calculations performed for various $(\overline{\mathrm{Ri}}, \omega)$ in which the growth rate $\lambda_{r}$ is plotted as a function of $\left(k, m_{0}\right)$. The patterns realized in all configurations are qualitatively similar, taking the form of narrow rays emanating from the point $\left(k, m_{0}\right)=(0,0)$. This pattern indicates that the relation $\lambda_{r}\left(k, m_{0}\right)$ can be more efficiently described in polar coordinates $(\kappa, \theta)$, which are defined as follows:

$$
k=\kappa \cos (\theta), \quad m_{0}=\kappa \sin (\theta) .
$$

Figure 3 presents the counterparts of the growth rate patterns in Fig. 2 that are plotted as functions of $(\kappa, \theta)$. The unstable regions take the form of vertical bands that are located in the vicinity of $\theta \approx \pi / 2$ and thus represent amplifying harmonics with almost horizontal wave fronts. The range of unstable wavenumbers in Fig. 3 is limited to $\kappa<\kappa_{\max } \sim 0.1$. In terms of dimensional wavelengths $L^{*}$, this condition translates to

$$
L^{*}>\frac{2 \pi}{\kappa_{\max }} d \sim 0.6 \mathrm{~m} .
$$

The stability of relatively short modes $\left(L^{*} \lesssim 0.6 \mathrm{~m}\right)$ is attributed to the action of molecular dissipation (viscosity and diffusivity), which preferentially affects small scales.

Another notable feature of patterns in Fig. 3, which substantially expedites our subsequent calculations, is the monotonic increase in the growth rate $\lambda_{r}$ with decreasing wavenumber $\kappa$ for any given $\theta$. While no formal proof of this property is available at this time, the inspection of numerous cases (only a small fraction of which is presented here) indicates that it is a generic feature of the oscillatory Couette flow. This finding implies that the maximal growth rate $\lambda_{\max }$ can be obtained by considering the long wavelength limit $(\kappa \rightarrow 0)$, in which case (20) reduces to:

$$
\lambda_{\max }=\max _{\theta}\left[\lim _{\kappa \rightarrow 0}\left(\lambda_{r}\right)\right] .
$$

Formulation (24) makes it possible to efficiently explore the wide range of governing parameters $(\overline{\mathrm{Ri}}, \omega)$ by assuming small finite wavenumber $\left(\kappa=10^{-6}\right.$ was used in the following calculation) and maximizing $\lambda_{r}$ over one-dimensional interval $0<\theta<\pi$ rather than over the entire two-dimensional space of $\left(k, m_{0}\right)$.

Figure 4 presents the resulting pattern of $\lambda_{\max }(\overline{\mathrm{Ri}}, \omega)$, which indicates that the growth rate monotonically increases with decreasing $\overline{\mathrm{Ri}}$ and with increasing $\omega$. The growth rate pattern is visibly different in the narrow region of relatively low Richardson numbers $(\overline{\mathrm{Ri}}<1.5)$, where decrease in $\lambda_{\max }(\overline{\mathrm{Ri}})$ is particularly rapid. In the rest of the domain, the variation of the growth rate with 

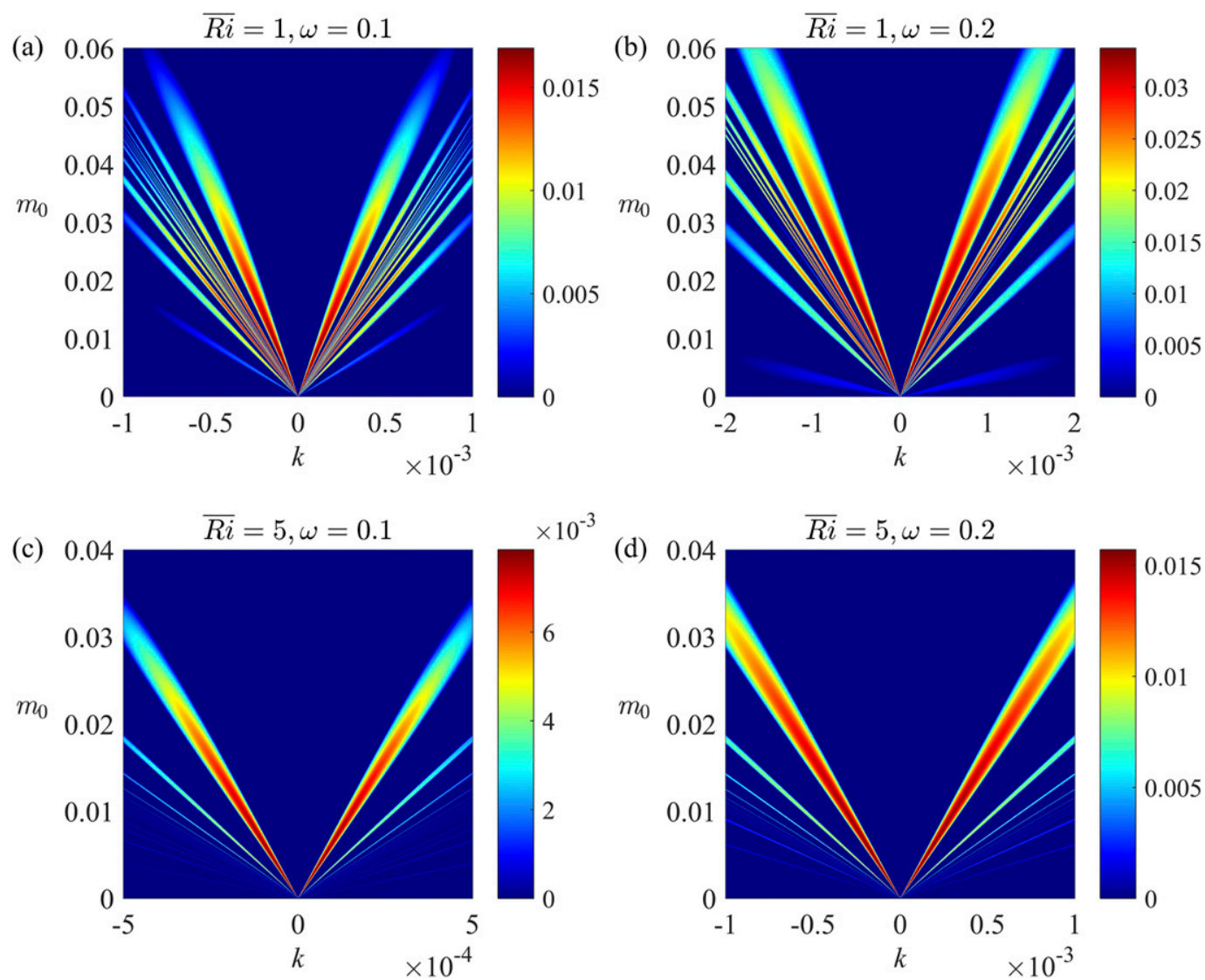

FIG. 2. The growth rate $\lambda_{r}$ is plotted as a function of wavenumbers $\left(k, m_{0}\right)$ for various combinations of the Richardson number and frequency: (a) $\overline{\mathrm{Ri}}=1, \omega=0.1$; (b) $\overline{\mathrm{Ri}}=1, \omega=0.2$; (c) $\overline{\mathrm{Ri}}=5, \omega=0.1$; and (d) $\overline{\mathrm{Ri}}=5, \omega=0.2$. Only positive growth rates are shown. The maximal growth rate increases with increasing frequency, whereas the increase in $\overline{\mathrm{Ri}}$ has an adverse effect on the instability.

both frequency and Richardson number are more gradual. In particular, the $\lambda_{\max }(\omega)$ relation for $\overline{\mathrm{Ri}}>1.5$ is well described by the proportionality $\lambda_{\max } \propto \omega$, as apparent from Fig. 5a. This property can be rationalized by the following argument. The calculation in Fig. 4 is based on the limit $\kappa \rightarrow 0$, which represents relatively large spatial scales. On such scales, the direct influence of molecular dissipation is expected to be negligible and the linear system (13) reduces to

$$
\left\{\begin{array}{l}
\frac{\partial \rho^{\prime}}{\partial t}+a_{U} \cos (\omega t) z \frac{\partial \rho^{\prime}}{\partial x}-\frac{\partial \psi^{\prime}}{\partial x}=0 \\
\frac{\partial \nabla^{2} \psi^{\prime}}{\partial t}+a_{U} \cos (\omega t) z \frac{\partial \nabla^{2} \psi^{\prime}}{\partial x}+\operatorname{Pr} \frac{\partial \rho^{\prime}}{\partial x}=0
\end{array} .\right.
$$

To explore properties of this nondissipative system, we consider the transformation

$$
\left\{\begin{array}{l}
t \rightarrow C t, x \rightarrow C x, z \rightarrow C z \\
\psi^{\prime} \rightarrow C^{2} \psi^{\prime}, \rho^{\prime} \rightarrow \rho^{\prime} \\
\omega \rightarrow C^{-1} \omega
\end{array}\right.
$$

and focus on $C \ll 1$, which represents an increase in the frequency of oscillations relative to some nominal configuration. As a result, (25) further reduces to

$$
\left\{\begin{array}{l}
\frac{\partial \rho^{\prime}}{\partial t}+a_{U} \cos (\omega t) z \frac{\partial \rho^{\prime}}{\partial x}=0 \\
\frac{\partial \nabla^{2} \psi^{\prime}}{\partial t}+a_{U} \cos (\omega t) z \frac{\partial \nabla^{2} \psi^{\prime}}{\partial x}+\operatorname{Pr} \frac{\partial \rho^{\prime}}{\partial x}=0
\end{array},\right.
$$

which is invariant with respect to the transformation (26). This invariance implies that the increase in frequency is associated with the equivalent reduction of spatial and temporal scales. The growth rate is inversely proportional to temporal scales, and therefore it should be directly proportional to the background oscillation frequency. Thus, for sufficiently large frequencies, $\lambda_{\max }$ is expected to be well described by the relation

$$
\lambda_{\max }=\lambda_{\text {norm }}(\overline{\mathrm{Ri}}) \omega .
$$



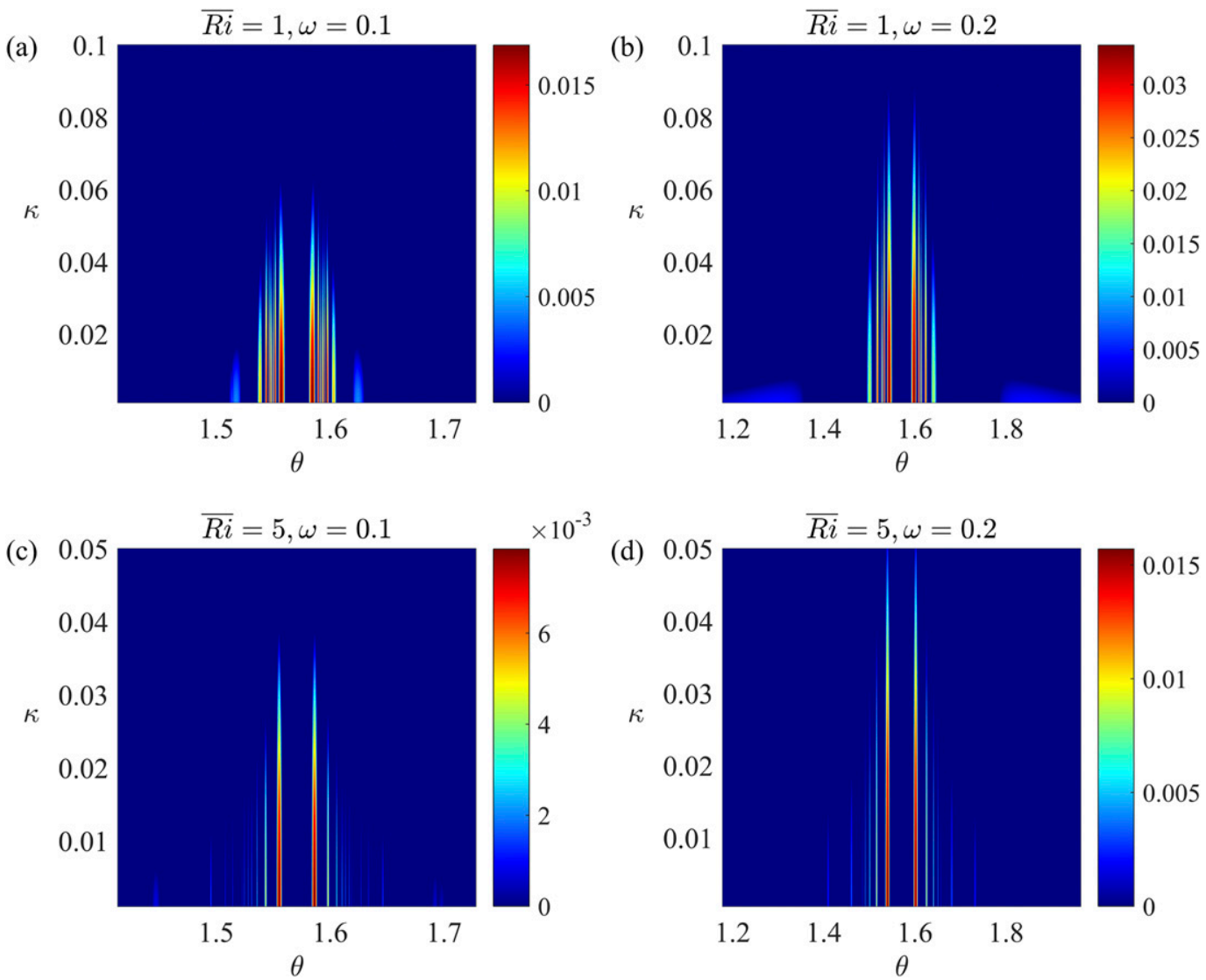

FIG. 3. As in Fig. 2, but the growth rate pattern is now presented in polar coordinates $(\kappa, \theta)$, which are defined in (22). Note the monotonic increase in $\lambda_{r}$ with decreasing $\kappa$.

This expectation is readily confirmed by the diagnostics in Fig. 5b, which plots the ratios $\lambda_{\max } / \omega$ as a function of $\overline{\mathrm{Ri}}$ for 23 values of $\omega$, spanning the interval from 0.15 to 1. Remarkably, all 23 curves in Fig. 5 b are visually indistinguishable from each other.

The dependence of $\lambda_{\text {norm }}$ on $\overline{\mathrm{Ri}}$ is well described by an empirical relation

$$
\lambda_{\text {norm }}=\frac{c_{1}}{\sqrt{\overline{\mathrm{Ri}}}}+\frac{c_{2}}{\overline{\mathrm{Ri}}},
$$

where $\left(c_{1}, c_{2}\right)$ are obtained from the best fit of (29) to the pattern in Fig. 5 b:

$$
c_{1}=0.3553, \quad c_{2}=-0.01467 .
$$

The proposed empirical model retains its form after conversion to the dimensional units:

$$
\lambda_{\max }^{*}=\left(\frac{c_{1}}{\sqrt{\mathrm{Ri}}}+\frac{c_{2}}{\overline{\mathrm{Ri}}}\right) \omega^{*} .
$$

The relative RMS difference between the raw results in Fig. 4 and (31) is less than $0.6 \%$ when evaluated over the range $1.5<\overline{\mathrm{Ri}}<10$. Therefore, relation (31) can be used to estimate the growth rate of time-dependent shears for given environmental conditions.

To glean some insight into the potential significance of TDSI, it is desirable to assess its strength in the context of other destabilizing processes that occur in oceanic shear flows. This task can be accomplished, for instance, by comparing the TDSI growth rates with those of KHI. The intensity of KHI is dependent on the Richardson number and on the assumed basic velocity and density profiles. However, the calculations performed for the harmonic (Balmforth and Young 2002) and hyperbolic tangent (Hazel 1972) shears indicate that, for moderately subcritical Richardson numbers $(\mathrm{Ri} \sim 0.1)$, the KHI growth rates are on the order of

$$
\lambda_{\mathrm{KHI}}^{*} \sim 0.1 \frac{\partial \bar{u}^{*}}{\partial z^{*}} .
$$

This crude estimate provides us with a convenient reference point for interpreting the results in Fig. 4. Therefore, in Fig. 6, we plot the pattern of growth rates normalized by the RMS basic shear: 


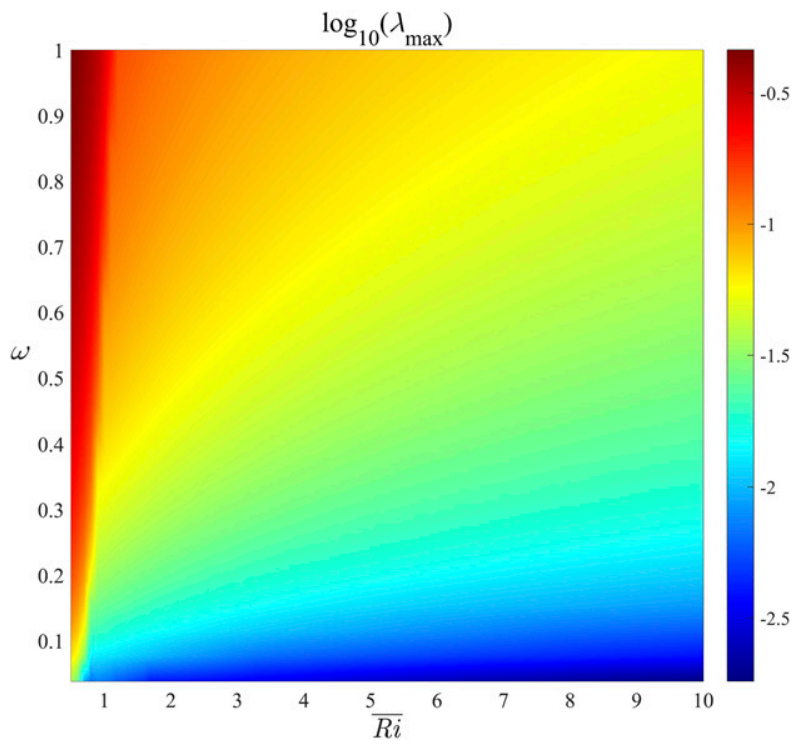

FIG. 4. The decimal logarithm of the maximal growth rate $\lambda_{\max }$ is plotted as a function of $(\overline{\mathrm{Ri}}, \omega)$.

$$
\lambda_{S}=\frac{\lambda_{\max }^{*}}{\sqrt{\left[\left(\frac{\partial \bar{u}^{*}}{\partial z^{*}}\right)^{2}\right]_{t}}}=\lambda_{\max } \sqrt{\frac{\overline{\mathrm{Ri}}}{\operatorname{Pr}}} .
$$

In the majority of the parameter space in Fig. 6, the ratios of the growth rate and shear are contained within the range of $0.01<\lambda_{S}<0.1$. This finding implies that, in terms of its ability to rapidly extract the energy from the basic shear, TDSI is somewhat less effective than KHI $\left(\lambda_{S \mathrm{KHI}} \sim 0.1\right)$ but not dramatically so.

\section{Physical interpretation}

In an attempt to identify the essential physics of TDSI, the governing equations in (13) are reduced to

$$
\left\{\begin{array}{l}
\frac{D \rho^{\prime}}{D t}=w^{\prime} \\
\frac{D \varsigma^{\prime}}{D t}=-\operatorname{Pr} \frac{\partial \rho^{\prime}}{\partial x}
\end{array}\right.
$$

where $\varsigma^{\prime}=\nabla^{2} \psi^{\prime}$ is the vorticity perturbation, all dissipative processes are neglected, and $D / D t$ represents the linearized material derivative:

$$
\frac{D}{D t} \equiv \frac{\partial}{\partial t}+\bar{u} \frac{\partial}{\partial x}
$$

In the absence of shear, system (34) supports regular plane-wave solutions that maintain their amplitude in time. The physics of internal waves is well understood
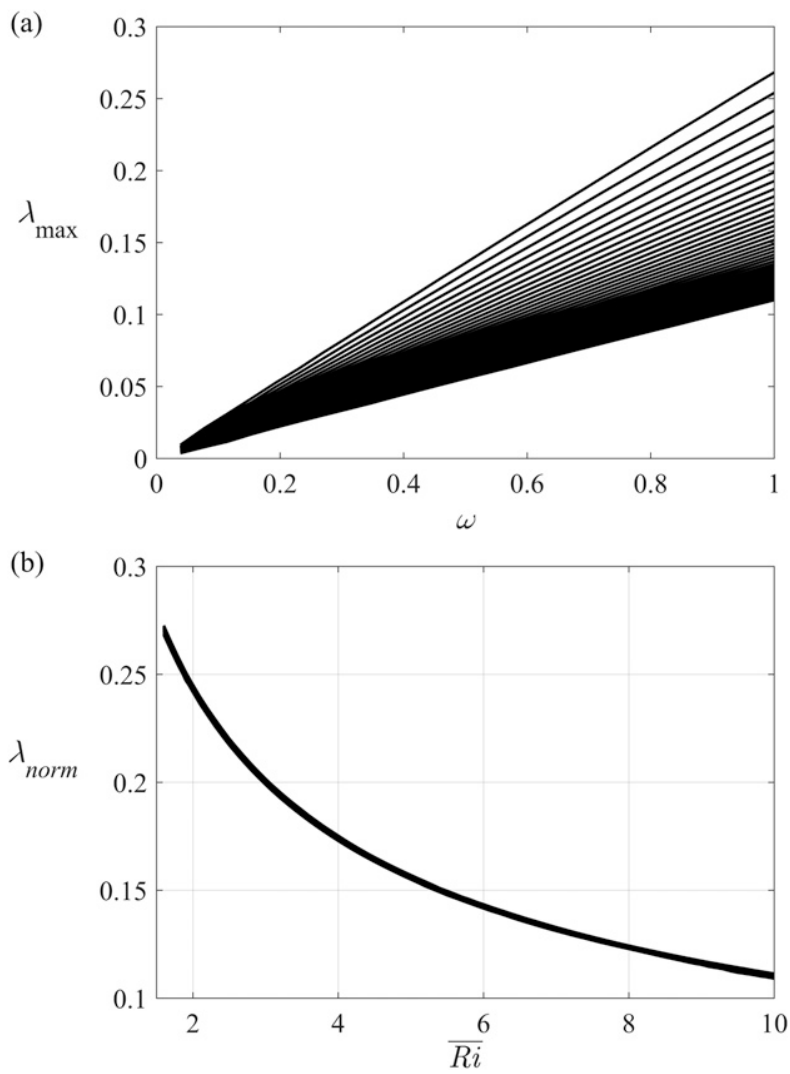

FIG. 5. (a) The maximal growth rate $\lambda_{\max }$ is plotted as a function of frequency $\omega$ for various values of the Richardson number in the range $1.6 \leq \overline{\mathrm{Ri}} \leq 10$ with increments of 0.2 . The growth rate monotonically decreases with the increasing Richardson number. (b) The normalized growth rate $\lambda_{\text {norm }}=\lambda_{\max } \omega^{-1}$ is plotted as a function of the Richardson number for various values of frequency number in the range $0.15<\omega \leq 1$ with increments of 0.0385 .

and it is illustrated in Fig. 7. The upper schematic represents the evolution of a harmonic perturbation that displaces the density interface downward in some regions (e.g., point $\mathrm{A}_{1}$ ) and upward in others (e.g., point $A_{3}$ ). The response of the system to this perturbation is apparent from the vorticity equation in (34). The tilt of the density interface $\left[\left(\partial \rho^{\prime} / \partial x\right)>0\right]$ at point $\mathrm{A}_{2}$ results in the clockwise torque $\left[\left(D \varsigma^{\prime} / D t\right)>0\right]$, which reflects the tendency of buoyancy forces to flatten inclined density interfaces. This torque acts in the manner opposing the initial displacement, thereby providing the restoring force that eventually reverses the tilt of the density interface. This process then repeats over and over, which maintains the oscillatory motion of invariable magnitude.

Note that the dynamics of buoyancy-driven oscillations illustrated in the upper panel of Fig. 7 pertains to both vertically sheared and uniform flows. The essential difference between the two systems comes into play by 


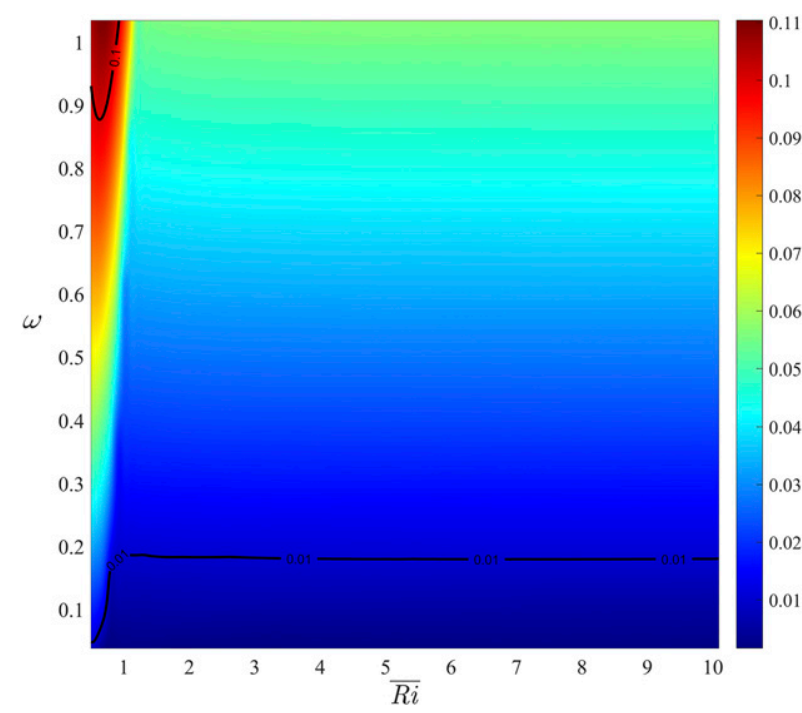

FIG. 6. The ratio of the maximal growth rate and the mean shear $\left(\lambda_{S}=\lambda_{\max } / S\right)$ is plotted as a function of $(\overline{\mathrm{Ri}}, \omega)$. Most of the data are in the range of moderate growth rates $0.01<\lambda_{S}<0.1$.

considering effects of strain produced by the basic Couette flow on the harmonic perturbation. The oscillation of basic shear leads to the corresponding periodic variation in the orientation of the perturbation wave fronts. The lower panel (Fig. 7) illustrates the system evolution during the phase when the perturbation is oriented in the direction opposing the background shear. In this regime, the basic shear flow tends to make the perturbation wave fronts more vertical. Such a change in the orientation of wave fronts has an interesting consequence of amplifying the vertical velocity component-the kinematic Orr effect (e.g., Lindzen 1988). This effect can be illustrated by relating $w^{\prime}=$ $\partial \psi^{\prime} / \partial x$ to $\varsigma^{\prime}=\nabla^{2} \psi^{\prime}$ as follows:

$$
\nabla^{2} w^{\prime}=\frac{\partial s^{\prime}}{\partial x}
$$

Assuming the normal mode solutions for $w^{\prime}$ and $\varsigma^{\prime}$ :

$$
\left\{\begin{array}{c}
\varsigma^{\prime}=\hat{s} \cos (k x+m z) \\
w^{\prime}=\hat{w} \sin (k x+m z)
\end{array},\right.
$$

we arrive at

$$
\hat{w}=\frac{k \hat{s}}{k^{2}+m(t)^{2}} .
$$

Thus, for any given amplitude of vorticity $\hat{\boldsymbol{s}}, \hat{w}$ tends to increase with decreasing $m^{2}$, which happens when wave fronts become more upright. Now let us consider for a moment a configuration where this strain-induced increase in vertical velocity occurs in upwelling locations-such as the point $\mathrm{A}_{3}$ in the upper panel of Fig. 7. In this case, the perturbation will gain energy and its amplitude will increase. In order for this mechanism to produce persistent long-term growth, the periodicity of the buoyancy-driven oscillating mode should match the periodicity of strain-induced forcing. The maximum of $\hat{w}$ corresponds to the minimum of $m^{2}$ which can be achieved either once or twice per shear period, depending on relative values of $m_{0}$ and $A_{u}$. For instance, in the case presented in Fig. 1, the dominant period of the perturbation is twice that of the background shear. Thus, this realization of TDSI can be placed into a broader class of parametric subharmonic instabilities of time-dependent flows (e.g., Drazin and Reid 1981).

Of course, the mechanism illustrated in Fig. 7 demands that the strain-induced upwelling occurs in regions where the motion of buoyancy-driven oscillatory mode is also upward for much of the oscillation period and vice versa. One can easily imagine configurations in which it is not the case. For instance, if these two components of vertical velocity are anticorrelated, then the buoyancy-driven oscillatory mode would decay rather than grow. The phase difference between the strain-induced velocity forcing and the perturbation pattern is ultimately set by the initial value of the vertical wavenumber $\left(m_{0}\right)$ or - to be more precise-by the initial inclination of wave fronts, as measured by $m_{0} / k$. For some values of $m_{0}$, the strain-induced forcing is stabilizing but for others it is destabilizing. This sensitivity of the stability/instability of individual harmonics to $m_{0}$ is reflected very clearly in the growth rate patterns $\lambda_{r}\left(k, m_{0}\right)$, such as shown in Fig. 2. Nevertheless, it should be kept in mind that the overall instability of any system is ultimately controlled by its most unstable component. Therefore, the mechanism of TDSI is based fundamentally on the emergence of unstable harmonics in an oscillating shear for certain values of $m_{0}$.

\section{Three-dimensional model}

The foregoing analysis of time-dependent shear instability in $2 \mathrm{D}$ provides essential guidance for the exploration of a more realistic and challenging threedimensional case. At the same time, the extension of our analysis into $3 \mathrm{D}$ makes it possible to incorporate the effects of planetary rotation, which two-dimensional models cannot represent. In particular, the background shear associated with internal waves now acquires shear components in both $x$ and $y$ directions:

$$
\left\{\begin{array}{l}
A_{U}=a_{U} \sin (\omega t) \\
A_{V}=a_{V} \cos (\omega t)
\end{array} .\right.
$$



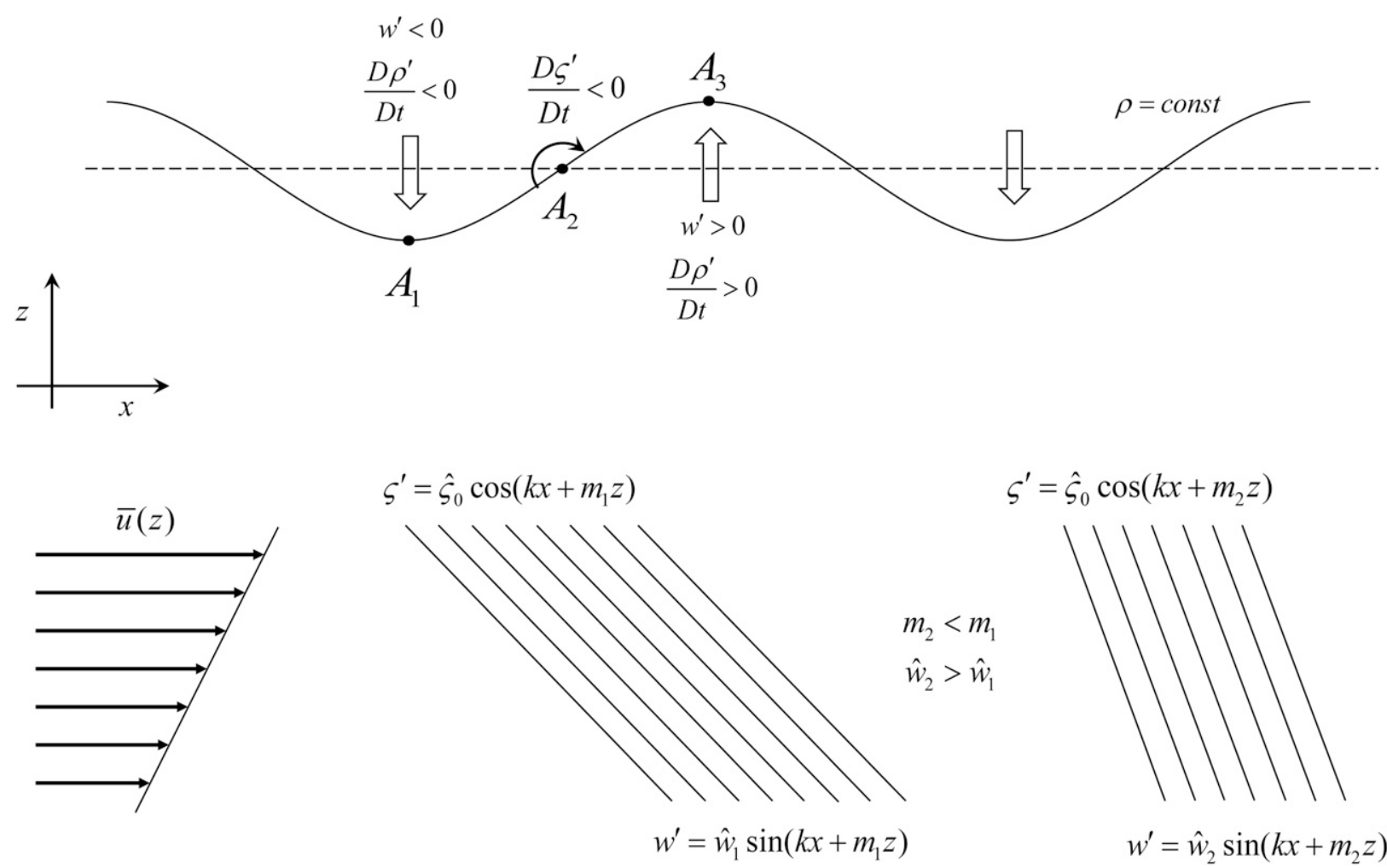

$$
\varsigma^{\prime}=\hat{\varsigma}_{0} \cos \left(k x+m_{2} z\right)
$$

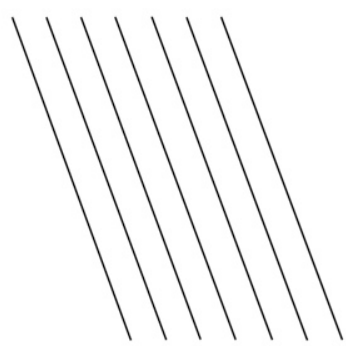

$$
w^{\prime}=\hat{w}_{2} \sin \left(k x+m_{2} z\right)
$$

FIG. 7. Schematic diagram illustrating the physical mechanism of TDSI.

As shown in the appendix, the amplitudes of shear components $\left(a_{U}, a_{V}\right)$ are determined by the mean Richardson number and the wave frequency as follows:

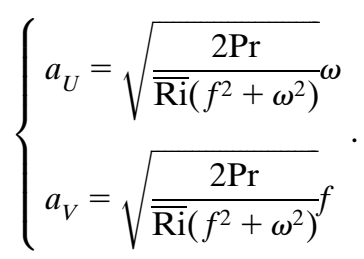

As previously (section 3 ), the growth rates were calculated by integrating the ODE system (12) in time and using the best linear fit to $(1 / 2) \ln (e)$, where the net perturbation energy $e$ in 3D takes the form

$$
e=\frac{|\hat{u}|^{2}+|\hat{v}|^{2}+|\hat{w}|^{2}}{4}+\frac{\operatorname{Pr}|\hat{\rho}|^{2}}{4}
$$

Taking planetary rotation into account makes the shear instability problem dynamically richer and more interesting. Figure 8 presents typical patterns the growth rate $\lambda_{r}$ as a function of $(k, l)$ for various values of $(\overline{\mathrm{Ri}}, \omega)$ and $m_{0}=0.01$. The three-dimensional visualization of a typical $\lambda_{r}\left(k, l, m_{0}\right)$ relation is shown in Fig. 9, revealing a rather intricate growth rate pattern realized in the presence of planetary rotation.
The principal complication in the $3 \mathrm{D}$ case is that the calculation of largest growth rates $\lambda_{\max }$ now requires maximization of $\lambda_{r}$ over the three-dimensional wavenumber space $\left(k, l, m_{0}\right)$. This difficulty, however, is alleviated by adopting the spherical coordinate system $(\kappa, \theta, \gamma)$ defined as follows:

$$
\begin{aligned}
k & =\kappa \cos (\theta) \cos (\gamma), \quad l=\kappa \cos (\theta) \sin (\gamma), \\
m_{0} & =\kappa \sin (\theta) .
\end{aligned}
$$

In all cases considered, we find that $\lambda_{r}$ monotonically increases with decreasing $\kappa$, which implies that the maximal growth rate can be obtained using

$$
\lambda_{\max }=\max _{\theta, \gamma}\left[\lim _{\kappa \rightarrow 0}\left(\lambda_{r}\right)\right] .
$$

This simplification allows us to proceed in the manner analogous to that adopted for the two-dimensional case (section 3). We assume small finite wavenumber $\left(\kappa=10^{-6}\right)$ and maximize $\lambda_{r}$ over the two-dimensional interval $0<\theta<\pi, 0<\gamma<2 \pi$. The results are shown in Fig. 10, where we plot the growth rates $\lambda_{\max }$ as a function of frequency $\omega$ for $\overline{\mathrm{Ri}}=1$ and $\overline{\mathrm{Ri}}=5$. As previously (section 3), we find that $\lambda_{\max }$ monotonically increases with increasing $\omega$. The growth rates realized at the larger 
(a)

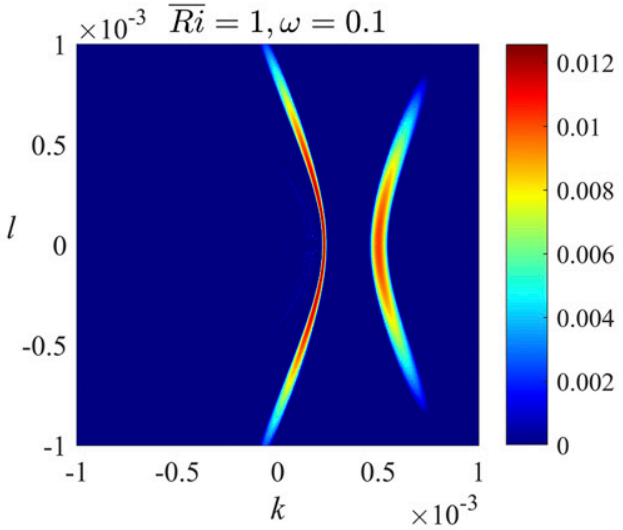

(c)

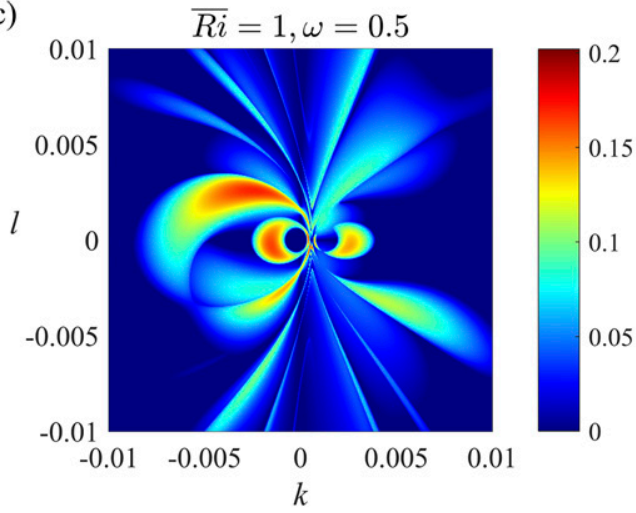

(b)

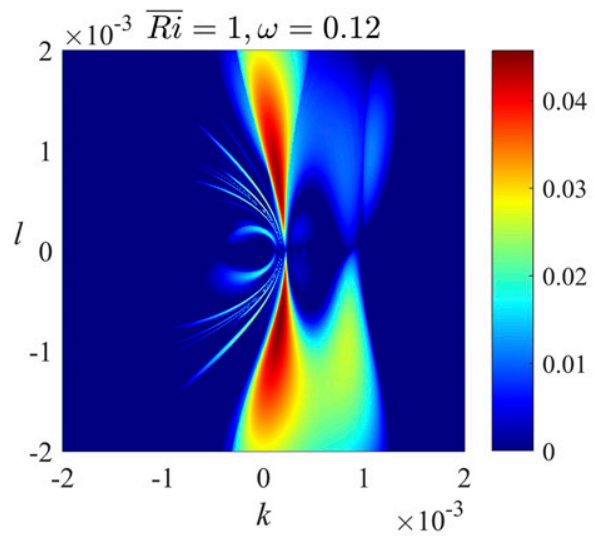

(d)

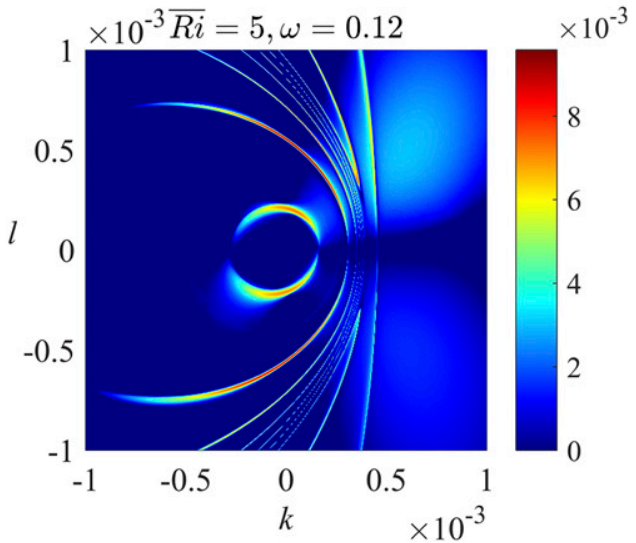

FIG. 8. The growth rate $\lambda_{r}$ is plotted as a function of horizontal wavenumbers $(k, l)$ for $m_{0}=0.01, f=0.1$, and various combinations of the Richardson number and frequency: (a) $\overline{\mathrm{Ri}}=1, \omega=0.1$; (b) $\overline{\mathrm{Ri}}=1, \omega=0.12$; (c) $\overline{\mathrm{Ri}}=1, \omega=0.5$; and (d) $\overline{\mathrm{Ri}}=5, \omega=0.12$. Only positive growth rates are shown.

Richardson number $(\overline{\mathrm{Ri}}=5)$ are significantly lower than those for $\overline{\mathrm{Ri}}=1$. Also suggestive is the comparison of $3 \mathrm{D}$ growth rates with the corresponding $2 \mathrm{D}$ calculations, which are represented in Fig. 9 by dashed curves. While $3 \mathrm{D}$ and $2 \mathrm{D}$ results are close for relatively rapid oscillations $(\omega \geq 0.2)$, significant differences are apparent at near-inertial frequencies $(\omega \approx 0.1)$. The latter observation is readily confirmed by inspecting the $\lambda(\overline{\mathrm{Ri}})$ relations for $\omega=0.1$ and $\omega=0.2$ in Fig. 11. For $\omega=0.2$, the $2 \mathrm{D}$ and $3 \mathrm{D}$ patterns are generally similar, with $3 \mathrm{D}$ growth rates exceeding the $2 \mathrm{D}$ ones by $20 \%-30 \%$. The inertial shear case, on the other hand, is marked by cardinal dissimilarities between the $2 \mathrm{D}$ and $3 \mathrm{D}$ solutions-the growth rates in 3D are now less, by an order of magnitude, than the corresponding $2 \mathrm{D}$ values.

The differences between 2D and 3D stability characteristics at low frequencies can be attributed to (i) the influence of planetary rotation on the temporal pattern of the background shear in (40) and/or (ii) the direct effects of the Coriolis parameter in the perturbative system (6). To glean some insight into the relative significance of these effects, a series of calculations in
Fig. 11 were reproduced using a hybrid model, which retains the Coriolis parameter in the calculation of basic shear in (40) while neglecting planetary rotation in the perturbative system (6). These calculations, which are also included in Fig. 11, indicate that much of the destabilizing influence of planetary rotation can be ascribed to its direct effect on the perturbative system. This influence can be substantial and, somewhat unexpectedly, it can be either destabilizing ( $\omega=0.1$ case) or stabilizing ( $\omega=0.2$ calculation).

The significance of taking into account the Coriolis effect in stability analyses of (6) is revealed perhaps most clearly by the dissimilar growth patterns $\lambda_{r}(k, l)$ realized in rotating and hybrid models, such as those shown in Fig. 12. The original model, which fully accounts for planetary rotation, produces the "tropical butterfly" pattern (Fig. 12a). The "alien spider" structure in Fig. 12b was obtained using the hybrid model that ignores the Coriolis effect in the perturbative system (6). Both tropical butterfly and alien spider patterns are remarkably intricate, visually striking, and rich in fine details. This complexity is surprising, given the minimal 


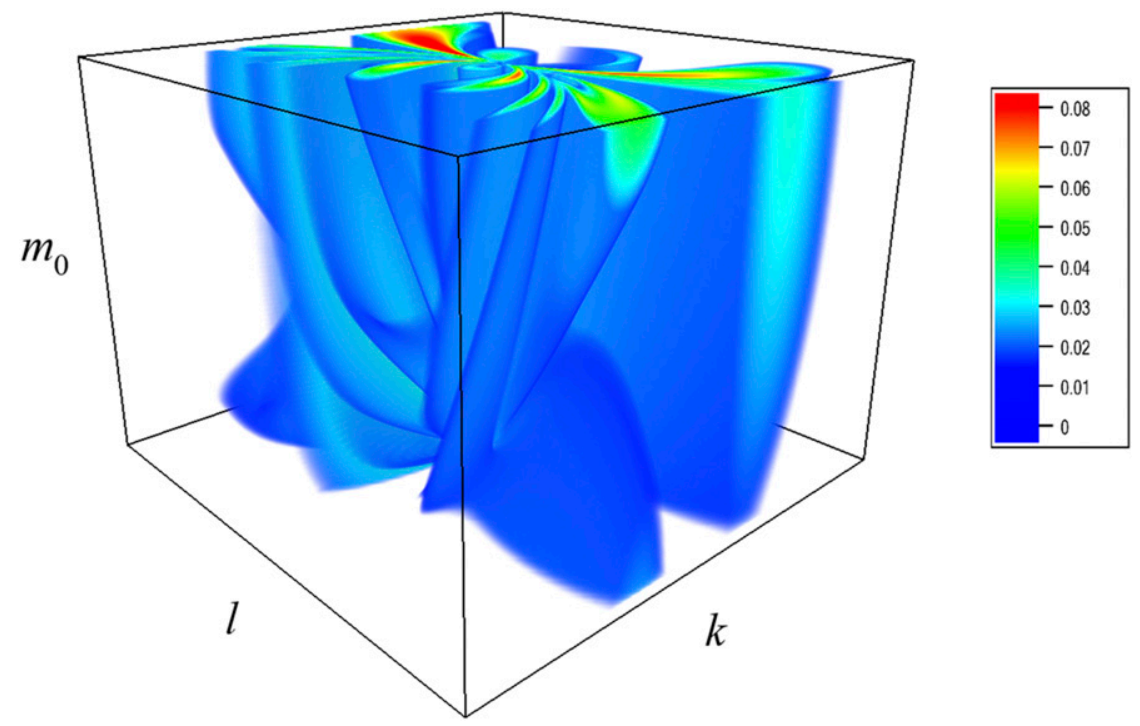

FIG. 9. Three-dimensional visualization of the growth rate $\lambda_{r}$ as a function of $\left(k, l, m_{0}\right)$ in the domain $-3 \times 10^{-3}<k<3 \times 10^{-3},-3 \times 10^{-3}<l<3 \times 10^{-3}$, and $0<m_{0}<0.01$.

character of systems being considered in the present investigation. While the maximal growth rates in Figs. $12 \mathrm{a}$ and $12 \mathrm{~b}$ differ by a mere $\sim 30 \%$, the Coriolis effect completely changes the symmetry characteristics of the growth rate patterns. The alien spider (Fig. 12b) is perfectly symmetric relative to the $k$ axis, which is oriented in the propagation direction of the basic internal wave (the appendix). This symmetry is visibly violated in the tropical butterfly pattern, implying that the growth rate relation in Fig. 12a is no longer invariant with respect to $l \rightarrow-l$ transformation.

\section{Discussion}

This study presents the stability analysis of spatially uniform but time-dependent vertical shears in two and three dimensions. The overarching conclusion that permeates all aspects of our investigation is that of a profound influence of temporal variability of shear flows on their stability. The extensive exploration of the parameter space, which spans a wide range of frequencies and Richardson numbers, reveals that temporally varying shears are unconditionally unstable. In this sense, the steady basic state model, commonly used in stability analyses of parallel flows, can be viewed as a singular limit of corresponding time-dependent systems.

The growth rates realized in our model for moderately supercritical Richardson numbers $(\overline{\mathrm{Ri}} \sim 1)$ are less than, but comparable to, those expected for KelvinHelmholtz instabilities of steady shears. They monotonically increase with the increasing frequency of the background oscillations and decrease with the increasing Richardson number. The growth rates predicted by the three-dimensional model (section 4), which takes planetary rotation into account, are close to the corresponding two-dimensional (nonrotating) estimates for moderate and high frequencies $\left(\omega^{*}>2 f^{*}\right)$. In this regime, the growth rates are accurately represented by the explicit semiempirical expression (31). For near-inertial $\left(\omega^{*} \approx f^{*}\right)$ oscillations, on the other hand, the two-dimensional estimates significantly exceed their three-dimensional counterparts.

The proposed physical interpretation of TDSI is based on the strain-induced modulation of vertical velocity, commonly referred to as the Orr effect. The unstable TDSI modes can be described as plane internal waves that are resonantly forced by time-dependent basic

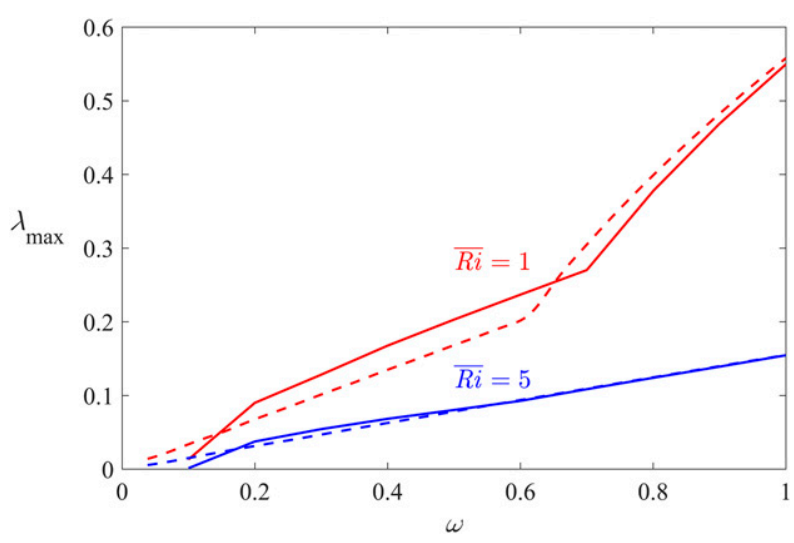

FIG. 10. The maximal growth rate $\lambda_{\max }$ is plotted as a function of frequency $\omega$ for $\overline{\mathrm{Ri}}=1$ (red) and $\overline{\mathrm{Ri}}=5$ (blue). The 3D and $2 \mathrm{D}$ results are indicated by the solid and dashed curves, respectively. 


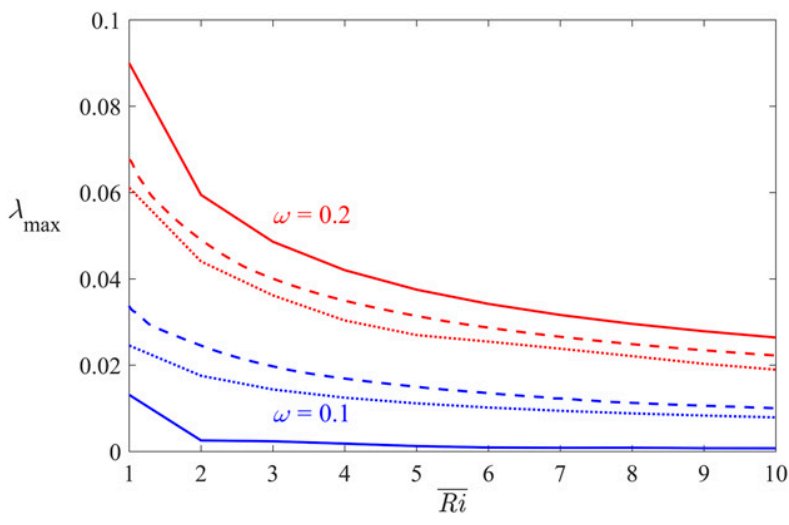

FIG. 11. The maximal growth rate $\lambda_{\max }$ is plotted as a function of the Richardson number ( $\overline{\mathrm{Ri}})$ for $\omega=0.1$ (blue) and $\omega=0.2$ (red). The $3 \mathrm{D}$ and $2 \mathrm{D}$ results are indicated by the solid and dashed curves, respectively. The dotted curves represent the hybrid model, which retains the Coriolis effect in the calculation of basic shear in (40) while neglecting it in the perturbative system (6).

shear flow. The Orr effect modifies their dynamics by providing the energy input into the perturbation field during each oscillation cycle. This amplification necessarily requires appropriate phase alignment between the strain-induced forcing and the perturbation pattern, which, in turn, is realized for proper initial orientations of the perturbation wave fronts.

In terms of underlying physics of TDSI, the present model should be contrasted with the stability analysis of free oscillatory flows characterized by solid-body rotation in the vertical plane (Majda and Shefter 1998, 2000). While the latter "rocking vorticity" basic states also exhibit instability for arbitrary large Richardson numbers, their dynamics are substantially different. In particular, the strain - the essential destabilizing ingredient of the time-dependent Couette model-plays no role in the instability of solutions analyzed by Majda and Shefter (1998). On the contrary, adding finite strain to the rocking vorticity patterns always makes them linearly stable. Of course, both time-dependent Couette and rocking vorticity models are interesting and bring complementary insights into shear flow instability. However, it should be kept in mind that oceanic shears are, by and large, associated with an active internal wave field. The spectrum of large-scale internal waves is dominated by slow modes with nearly flat wave fronts, predominantly horizontal particle displacements, and finite strain. Therefore, it is our belief that typical oceanic conditions may be better represented by the timedependent Couette model.

The spatial scales of unstable TDSI modes are relatively large $\left(L_{z}^{*}>0.6 \mathrm{~m}\right.$ and $L_{x, y}^{*}>20 \mathrm{~m}$ for typical oceanic conditions), which places them in the category of (a)

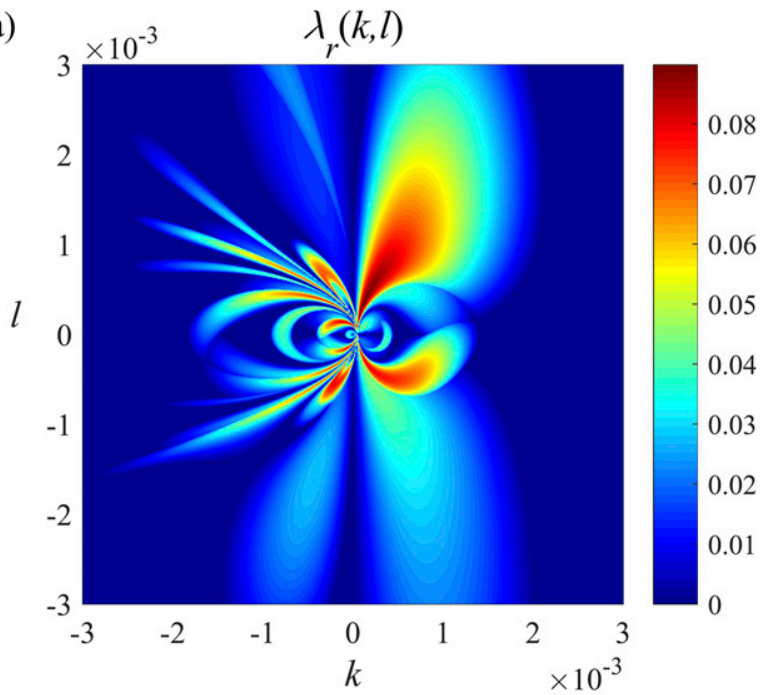

(b)

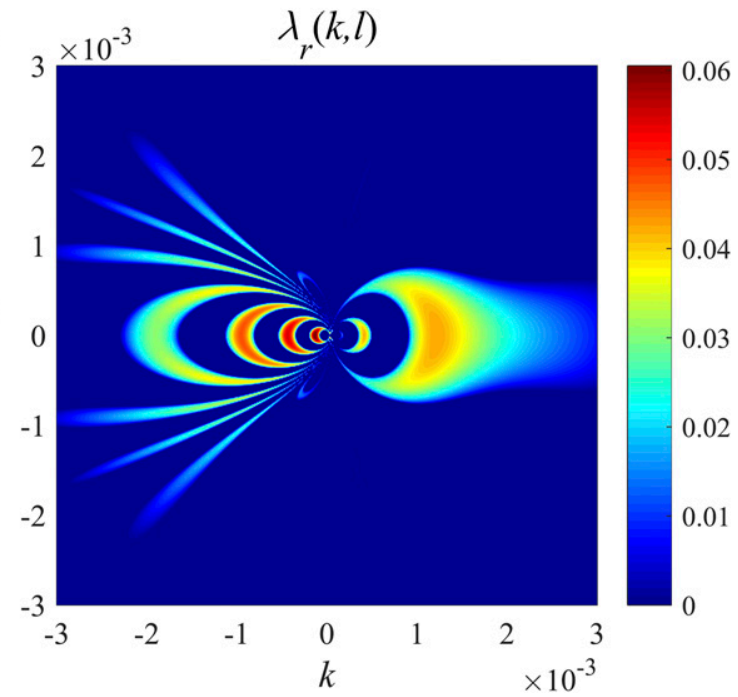

FIG. 12. The growth rate $\lambda_{r}$ is plotted as a function of horizontal wavenumbers $(k, l)$ for $\overline{\mathrm{Ri}}=1, \omega=0.2, m_{0}=0.002$, and $f=0.1$. (a) The experiment taking full account of planetary rotation. (b) The hybrid model that retains the Coriolis effect in the calculation of basic shear in (40) but neglects it in the perturbative system (6).

finescale phenomena. Therefore, the present linear model can offer only a limited insight into the ability of TDSI to induce irreversible mixing of seawater properties on the microscale. Nevertheless, it is plausible that the amplification of TDSI modes will ultimately create favorable conditions for either convective (top-heavy) or dynamic (Kelvin-Helmholtz) secondary instabilities, triggering the transition to fully developed turbulence. In this regard, TDSI could play a catalytic role in the cascade of energy and temperature variances to small scales, where they can be effectively removed by molecular dissipation. We also hypothesize that some TDSI 
modes could manifest themselves as layered patterns omnipresent in seismic images of oceanic fine structure (Holbrook et al. 2003; Ruddick et al. 2009). While such patterns are frequently and appropriately attributed (e.g., Biescas et al. 2008; Pinheiro et al. 2010) to thermohaline interleaving driven by doublediffusive convection (Stern 1967; Ruddick and Kerr 2003; Radko 2013) some seismic images could reflect finite-amplitude TDSI.

One of the distinguishing features of the present investigation is its framework, which assumes the basic states with spatially uniform shear. Aside from a series of analytical simplifications afforded by this configuration, it also helps us to clarify several aspects of the internal wave dynamics. In particular, the uniform gradient model excludes the possibility of destabilization through the resonant triad interaction, which is often invoked in stability analyses of internal waves (e.g., Mied 1976; Lombard and Riley 1996). The present work, therefore, draws attention to the existence of alternative routes to instability and dissipation. Likewise, the uniform shear model has led us to the conclusion that the presence of inflection points in vertical velocity profiles may not be essential for triggering instabilities of large-scale internal waves. This finding is particularly suggestive in view of qualitative dissimilarities in the stability properties of inflected and noninflected shears, which have been reported for various models of parallel flows (Drazin and Reid 1981; Rayleigh 1880). For instance, steady uniform unbounded shears are stable regardless of how low the Richardson numbers are (Knobloch 1984), whereas the inflected shears are unstable for $\mathrm{Ri}<1 / 4$ (e.g., Hazel 1972). Considering the general tendency of inflected shears to be less stable than their noninflected counterparts, it is plausible that the estimates in our study can serve as a lower bound for the growth rates realized for more irregular shear patterns, such as expected to occur in the ocean.

This project can be further developed in a number of promising directions. For instance, it could prove beneficial to extend our linear stability analyses beyond the monochromatic shear model. The temporal pattern of the basic shear can be represented by a superposition of Fourier components conforming to the Garrett-Munk spectrum with random initial phase distribution (e.g., Radko et al. 2015). Such an investigation would help to answer intriguing questions with regard to the role of various temporal harmonics in triggering TDSI. Our results consistently indicate that high-frequency modes are more effective in destabilizing shear flows than low-frequency harmonics. The spectrum of internal waves, on the other hand, is dominated by slow, near-inertial oscillations (Garrett and Munk 1972).
Therefore, the relative significance of slow and fast spectral components in determining the stability properties of wave-induced shears is not clear a priori. The analysis of time-dependent inflected shears also represents a natural extension of the present investigation. Another potentially profitable avenue of exploration is modeling of the nonlinear evolution of TDSI and transition to turbulence. The assessment of the associated vertical fluxes of seawater properties is expected to improve our understanding of wave-induced mixing in the ocean.

Acknowledgments. The author thanks the editor Paola Cessi and the anonymous reviewers for helpful comments. Support of the National Science Foundation (Grant OCE 1756491) is gratefully acknowledged.

\section{APPENDIX}

\section{Internal Wave Model}

To make this article self-contained, we now review the linear theory of monochromatic internal waves, casting it in terms of nondimensional variables introduced in section 2. The governing Navier-Stokes equations are linearized with respect to the basic state at rest and nondimensionalized as follows:

$$
\left\{\begin{array}{l}
\frac{\partial \rho_{w}}{\partial t}-w_{w}=0 \\
\frac{\partial u_{w}}{\partial t}-f v_{w}=-\operatorname{Pr} \frac{\partial p_{w}}{\partial x} \\
\frac{\partial v_{w}}{\partial t}+f u_{w}=-\operatorname{Pr} \frac{\partial p_{w}}{\partial y} \\
\frac{\partial w_{w}}{\partial t}=-\operatorname{Pr}\left(\frac{\partial p_{w}}{\partial z}+\rho_{w}\right) \\
\frac{\partial u_{w}}{\partial x}+\frac{\partial v_{w}}{\partial y}+\frac{\partial w_{w}}{\partial z}=0
\end{array},\right.
$$

where the subscript $w$ is used to emphasize that the field variables in (A1) represent free large-scale internal waves rather than their small-scale instabilities. We also assume that the molecular dissipation is negligible on the scale of these waves and seek plane-wave solutions satisfying (A1) as follows:

$$
\left\{\begin{array}{l}
u_{w}=\hat{u}_{w} \cos \left(k_{w} x+m_{w} z-\omega t\right) \\
v_{w}=\hat{v}_{w} \sin \left(k_{w} x+m_{w} z-\omega t\right) \\
w_{w}=\hat{w}_{w} \cos \left(k_{w} x+m_{w} z-\omega t\right) \\
p_{w}=\hat{p}_{w} \sin \left(k_{w} x+m_{w} z-\omega t\right) \\
\rho_{w}=\hat{\rho}_{w} \cos \left(k_{w} x+m_{w} z-\omega t\right)
\end{array}\right.
$$


In the confines of the $f$-plane model, we can assume that the wave is propagating in the positive $x$ direction without loss of generality, which accounts for the absence of $y$ dependence in the assumed form (A2). When (A2) is substituted into the linear system (A1) and the amplitudes of $\hat{w}_{w}, \hat{p}_{w}$, and $\hat{\rho}_{w}$ are sequentially eliminated, we explicitly connect the amplitudes of horizontal velocity components as follows:

$$
\hat{v}_{w}=\hat{u}_{w} \frac{f}{\omega} .
$$

Equation (A3) makes it possible to express the local RMS shear $S$ in terms of $\hat{u}_{w}$ :

$$
S \equiv \sqrt{\left[\left(\frac{\partial u_{w}}{\partial z}\right)^{2}+\left(\frac{\partial v_{w}}{\partial z}\right)^{2}\right]_{t}}=\hat{u}_{w} m_{w} \sqrt{\frac{\omega^{2}+f^{2}}{2 \omega^{2}}} .
$$

The nondimensional shear $S$, in turn, is directly related to the mean Richardson number:

$$
\overline{\mathrm{Ri}} \equiv \frac{N^{*^{2}}}{S^{*^{2}}}=\frac{\operatorname{Pr}}{S^{2}} .
$$

Drawing together (A3)-(A5), we express the amplitudes $\left(\hat{u}_{w}, \hat{v}_{w}\right)$ in terms of $\overline{\mathrm{Ri}}$ :

$$
\left\{\begin{array}{l}
\hat{u}_{w}=\sqrt{\frac{2 \operatorname{Pr}}{\overline{\operatorname{Ri}}\left(f^{2}+\omega^{2}\right)}} \frac{\omega}{m_{w}} \\
\hat{v}_{w}=\sqrt{\frac{2 \operatorname{Pr}}{\overline{\operatorname{Ri}}\left(f^{2}+\omega^{2}\right)}} \frac{f}{m_{w}}
\end{array} .\right.
$$

Finally, (A6) is used to evaluate the vertical shear components:

$$
\left\{\begin{array}{l}
\frac{\partial u_{w}}{\partial z}=-\hat{u}_{w} m_{w} \sin \left(k_{w} x+m_{w} z-\omega t\right)=-\sqrt{\frac{2 \operatorname{Pr}}{\operatorname{Ri}\left(f^{2}+\omega^{2}\right)}} \omega \sin \left(k_{w} x+m_{w} z-\omega t\right) \\
\frac{\partial v_{w}}{\partial z}=\hat{v}_{w} m_{w} \cos \left(k_{w} x+m_{w} z-\omega t\right)=\sqrt{\frac{2 \operatorname{Pr}}{\overline{\operatorname{Ri}}\left(f^{2}+\omega^{2}\right)}} f \cos \left(k_{w} x+m_{w} z-\omega t\right)
\end{array} .\right.
$$

Recalling that solution (A7) represents a plane wave in an unbounded medium with uniform stratification, it becomes apparent that all spatial locations $(x, z)$ are equivalent in terms of shear characteristics (except for different phases). Thus, a model of temporal variability of the wave-induced shear can be constructed, without loss of generality, by assuming $(x, z)=(0,0)$, which reduces (A7) to (39) and (40). The latter system is used in section 4 to represent the temporal variation in basic shear associated with large-scale internal waves.

\section{REFERENCES}

Atsavapranee, P., and M. Gharib, 1997: Structures in stratified plane mixing layers and the effects of cross-shear. J. Fluid Mech., 342, 53-86, https://doi.org/10.1017/S0022112097005399.

Balmforth, N. J., and Y.-N. Young, 2002: Stratified Kolmogorov flow. J. Fluid Mech., 450, 131-167, https://doi.org/10.1017/ S0022111002006371.

Biescas, B., V. Sallares, J. L. Pelegri, F. Machin, R. Carbonell, G. Buffett, J. J. Danobeitia, and A. Calahorrano, 2008: Imagining meddy finestructure using multichannel seismic reflection data. Geophys. Res. Lett., 35, L11609, https://doi.org/ 10.1029/2008GL033971.

Caulfield, C. P., and W. R. Peltier, 2000: The anatomy of the mixing transition in homogeneous and stratified free shear layers. J. Fluid Mech., 413, 1-47, https://doi.org/10.1017/ S0022112000008284.
Drazin, P. G., 1977: On the instability of an internal gravity wave. Proc. Roy. Soc. London, 356, 411-432, https://doi.org/10.1098/ rspa.1977.0142.

—, and W. H. Reid, 1981: Hydrodynamic Stability. Cambridge University Press, 628 pp.

Dunkerton, T. J., 1997: Shear instability of internal inertia-gravity waves. J. Atmos. Sci., 54, 1628-1641, https://doi.org/10.1175/ 1520-0469(1997)054<1628:SIOIIG > 2.0.CO;2.

Garrett, C., and W. Munk, 1972: Space-time scales of ocean internal waves. Geophys. Fluid Dyn., 3, 225-264, https://doi.org/ 10.1080/03091927208236082.

Hazel, P., 1972: Numerical studies of the stability of inviscid stratified shear flows. J. Fluid Mech., 51, 39-61, https://doi.org/ 10.1017/S0022112072001065.

Holbrook, W. S., P. Paramo, S. Pearse, and R. Schmitt, 2003: Thermohaline fine structure in an oceanographic front from seismic reflection profiling. Science, 301, 821-824, https:// doi.org/10.1126/science.1085116.

Howard, L. N., 1961: Note on a paper of John W. Miles. J. Fluid Mech., 10, 509-512, https://doi.org/10.1017/S0022112061000317.

Ivey, G. N., K. B. Winters, and J. R. Koseff, 2008: Density stratification, turbulence, but how much mixing? Annu. Rev. Fluid Mech., 40, 169-184, https://doi.org/10.1146/ annurev.fluid.39.050905.110314.

Kaminski, A. K., and W. D. Smyth, 2019: Stratified shear instability in a field of pre-existing turbulence. J. Fluid Mech., 862, 639658, https://doi.org/10.1017/jfm.2018.973.

Kelvin, L., 1871: Influence of wind and capillarity on waves in water supposed frictionless. Mathematical and Physical Papers, Vol. 4, Cambridge University Press, 76-85. 
Klostermeyer, J., 1982: On parametric instabilities of finite amplitude internal gravity waves. J. Fluid Mech., 119, 367-377, https://doi.org/10.1017/S0022112082001396.

Knobloch, E., 1984: On the stability of stratified plane Couette flow. Geophys. Astrophys. Fluid Dyn., 29, 105-116, https:// doi.org/10.1080/03091928408248185.

Lindzen, R. S., 1988: Instability of plane parallel shear flow. Pure Appl. Geophys., 126, 103-121, https://doi.org/10.1007/ BF00876917.

Lombard, P. N., and J. R. Riley, 1996: Instability and breakdown of internal gravity waves. I. Linear stability analysis. Phys. Fluids, 8, 3271-3287, https://doi.org/10.1063/1.869117.

Majda, A. J., and M. G. Shefter, 1998: Elementary stratified flows with instability at large Richardson number. J. Fluid Mech., 376, 319-350, https://doi.org/10.1017/S0022112098003085.

$\ldots$, and — 2000: Nonlinear instability of elementary stratified flows at large Richardson number. Chaos, 10, 3-27, https:// doi.org/10.1063/1.166472.

Mied, R. P., 1976: The occurrence of parametric instabilities in finite amplitude internal gravity waves. J. Fluid Mech., 78, 763784, https://doi.org/10.1017/S0022112076002735.

Miles, J. W., 1961: On the stability of heterogeneous shear flows. J. Fluid Mech., 10, 496-508, https://doi.org/10.1017/ S0022112061000305.

Peltier, W. R., and C. P. Caulfield, 2003: Mixing efficiency in stratified shear flows. Annu. Rev. Fluid Mech., 35, 135-167, https://doi.org/10.1146/annurev.fluid.35.101101.161144.

Pinheiro, L. M., H. Song, B. Ruddick, J. Dubert, I. Ambar, K. Mustafa, and R. Bezerra, 2010: Detailed 2-D imaging of the Mediterranean outflow and meddies off W Iberia from multichannel seismic data. J. Mar. Syst., 79, 89-100, https://doi.org/ 10.1016/j.jmarsys.2009.07.004.

Radko, T., 2013: Double-Diffusive Convection. Cambridge University Press. 344 pp.

_ 2016: Thermohaline layering in dynamically and diffusively stable shear flows. J. Fluid Mech., 805, 147-170, https://doi.org/ 10.1017/jfm.2016.547.

_ 2019: Thermohaline-shear instability. Geophys. Res. Lett., 46, 822-832, https://doi.org/10.1029/2018GL081009.

__ , J. Ball, J. Colosi, and J. Flanagan, 2015: Double-diffusive convection in a stochastic shear. J. Phys. Oceanogr., 45, 3155-3167, https://doi.org/10.1175/JPO-D-15-0051.1.

Rayleigh, L., 1880: On the stability, or instability, of certain fluid motions. Proc. London Math. Soc., 11, 57-70, https://doi.org/ 10.1112/plms/s1-11.1.57.

Richardson, L. F., 1920: The supply of energy from and to atmospheric eddies. Proc. Roy. Soc. London, 97A, 354-373, https:// doi.org/10.1098/rspa.1920.0039.
Ruddick, B., and O. Kerr, 2003: Oceanic thermohaline intrusions: Theory. Prog. Oceanogr., 56, 483-497, https://doi.org/10.1016/ S0079-6611(03)00029-6.

— - H. Song, C. Dong, and L. Pinheiro, 2009: Water column seismic images as maps of temperature gradient. Oceanography, 22, 192-206, https://doi.org/10.5670/oceanog.2009.19.

Shepherd, T. G., 1985: Time development of small disturbances to plane Couette flow. J. Atmos. Sci., 42, 1868-1872, https://doi.org/10.1175/1520-0469(1985)042<1868:TDOSDT> 2.0.CO;2.

Smyth, W. D., and J. N. Moum, 2012: Ocean mixing by KelvinHelmholtz instability. Oceanography, 25, 140-149, https:// doi.org/10.5670/oceanog.2012.49.

__ J. Moum, and D. Caldwell, 2001: The efficiency of mixing in turbulent patches: Inferences from direct simulations and microstructure observations. J. Phys. Oceanogr., 31, 1969-1992, https://doi.org/10.1175/1520-0485(2001)031<1969: TEOMIT>2.0.CO;2.

Stern, M. E., 1960: The "salt-fountain" and thermohaline convection. Tellus, 12, 172-175, https://doi.org/10.3402/tellusa.v12i2.9378.

_ 1967: Lateral mixing of water masses. Deep-Sea Res., 14, 747753, https://doi.org/10.1016/S0011-7471(67)80011-1.

Thorpe, S. A., 1971: Experiments on instability and turbulence of stratified shear flows: Miscible fluids. J. Fluid Mech., 46, 299319, https://doi.org/10.1017/S0022112071000557.

— 2005: The Turbulent Ocean. Cambridge University Press, 484 pp.

— W. W. Smyth, and L. Li, 2013: The effect of small viscosity and diffusivity on the marginal stability of stably stratified shear flows. J. Fluid Mech., 731, 461-476, https://doi.org/10.1017/ jfm.2013.378.

Troy, C. D., and J. R. Koseff, 2005: The instability and breaking of long internal waves. J. Fluid Mech., 543, 107-136, https:// doi.org/10.1017/S0022112005006798.

van Haren, H., L. Gostiaux, E. Morozov, and R. Tarakanov, 2014: Extremely long Kelvin-Helmholtz billow trains in the Romanche Fracture Zone. Geophys. Res. Lett., 41, 8445-8451, https://doi.org/10.1002/2014GL062421.

von Helmholtz, H., 1868: Uber discontinuierliche FlussigkeitsBewegungen [On the discontinuous movements of fluids]. Monatsber. Koniglichen Preuss. Akad. Wiss. Berlin, 23, 215-228.

Winters, K. B., 2008: Growth of inertia-gravity waves in sheared inertial currents. J. Fluid Mech., 601, 85-100, https://doi.org/ 10.1017/S0022112008000621.

Woods, J. D., 1968: Wave-induced shear instability in the summer thermocline. J. Fluid Mech., 32, 791-800, https://doi.org/ 10.1017/S0022112068001035. 